\title{
The INPHARMA technique for pharmacophore mapping: A theoretical guide to the method
}

\author{
Julien Orts ${ }^{\mathrm{a}, \mathrm{b}}$, Christian Griesinger ${ }^{\mathrm{b}}$, Teresa Carlomagno ${ }^{\mathrm{a}, *}$ \\ ${ }^{a}$ European Molecular Biology Laboratory (EMBL), Structural and Computational Biology Unit, Meyerhofstrasse 1, 69117 Heidelberg, Germany \\ ${ }^{\mathrm{b}}$ Department of NMR-based Structural Biology, Max Planck Institute for Biophysical Chemistry, Am Fassberg 11, 37077 Göttingen, Germany
}

\section{A R T I C L E I N F O}

Article history:

Received 15 April 2009

Available online 12 June 2009

\section{Keywords:}

NMR

INPHARMA

NOE

Drug design

Pharmacophore

\begin{abstract}
A B S T R A C T
During the process of drug discovery, INPHARMA can be used to derive the structure of receptor/lead compound complexes binding to each other with a $K_{d}$ in the $\mu \mathrm{M}$ to $\mathrm{mM}$ range. To be successful, the methodology needs adjustment of various parameters that depend on the physical constants of the binding event and on the receptor size. Here we present a thorough theoretical analysis of the INPHARMA interligand NOE effect in dependence of experimental parameters and physical constants. This analysis helps the experimentalist to choose the correct experimental parameters and consequentially to achieve optimal performance of the methodology.
\end{abstract}

(ㄷ) 2009 Elsevier Inc. All rights reserved.

\section{Introduction}

In structure-based drug design a three-dimensional picture of the binding mode of known ligands to the target macromolecule is essential to the elaboration of a high affinity drug. When the complex cannot be crystallized, NMR can be used to obtain structural information in solution. However, the determination of a high resolution structure of the complex by NMR in solution is often limited by the availability and the physical properties of the target, which in many cases is either too large to be observed by NMR, too insoluble or not available with the necessary ${ }^{13} \mathrm{C} /{ }^{15} \mathrm{~N} /{ }^{2} \mathrm{D}$ labeling from expression systems.

For ligands that bind weakly to the target $\left(K_{d}\right.$ in the $\mu \mathrm{M}$ range) detailed structural information on the ligand bound conformation can be obtained by transferred-NOEs and transferred-CCR rates [1$6]$. This approach requires small quantities of unlabeled target (1$10 \mu \mathrm{M}$ solution) and an excess of ligand, is applicable to any complex independently of the size of the target macromolecule and does not require any isotope labeling scheme. However, as the resonances of the target macromolecule are not observed, the methodology does not provide any structural information on the geometry of the intermolecular interactions in the complex. Models of the ligand binding mode can be obtained from the apo-structure of the target macromolecule and the bound structure of the ligand by docking calculations. The docking models are ranked on the basis of the computed intermolecular interaction energy or with respect to experimental information, such as the ligand

\footnotetext{
* Corresponding author. Fax: +49 62213878519.

E-mail address: teresa.carlomagno@embl.de (T. Carlomagno).
}

binding epitope, obtained by the STD or WATER-LOGSY approaches [7-10]. However, this process mostly results in multiple models for the complex structure due to the lack of site-specific structural information.

To overcome this problem, we developed the INPHARMA methodology (Interligand Noes for PHARmacophore MApping) that allows mapping the structure of the binding pocket of a macromolecule on the NMR resonances of two competitively binding ligands [11-14]. The method is based on the observation of interligand, spin-diffusion mediated, transferred-NOE data, between two ligands $L_{1}$ and $L_{2}$, binding competitively and weakly to a macromolecular receptor $T$ (Fig. 1). During the mixing time of the NOESY experiment, $L_{1}$ binds to the receptor and its protons $\left(H_{L_{1}} \mathrm{~s}\right)$ transfer their magnetization to the receptor protons $\left(H_{T} \mathrm{~s}\right)$. During the same mixing time of the NOESY experiment $L_{1}$ dissociates from the receptor and $L_{2}$ binds. The magnetization that was transferred from $H_{L_{1}}$ to $H_{T}$ can now be transferred from $H_{T}$ to $H_{L_{2}}$. This leads to an intermolecular peak between $H_{L_{1}}$ and $H_{L_{2}}$ although $L_{1}$ and $L_{2}$ have never been close in space at any time during the NMR experiments. The NOE peak between $H_{L_{1}}$ and $H_{L_{2}}$ is a spin-diffusion mediated effect via the receptor proton $H_{T}$. Clearly this effect can only occur if $H_{L_{1}}$ and $H_{L_{2}}$ are both close to the proton $H_{T}$ in the two complexes $T L_{1}$ and $T L_{2}$, respectively. A number of such interligand NOEs define the relative orientation of the two ligands in the receptor binding pocket. The INPHARMA methodology belongs to the class of ligand-detected approaches (where the resonances of the target macromolecule are not seen in the spectrum), like transferredNOEs, transferred-CCR rates, STD and others $[1,2,5,7,15]$. The binding pocket of the target macromolecule is here indirectly mapped on the resonances of the two ligands. Due to the nature of the 

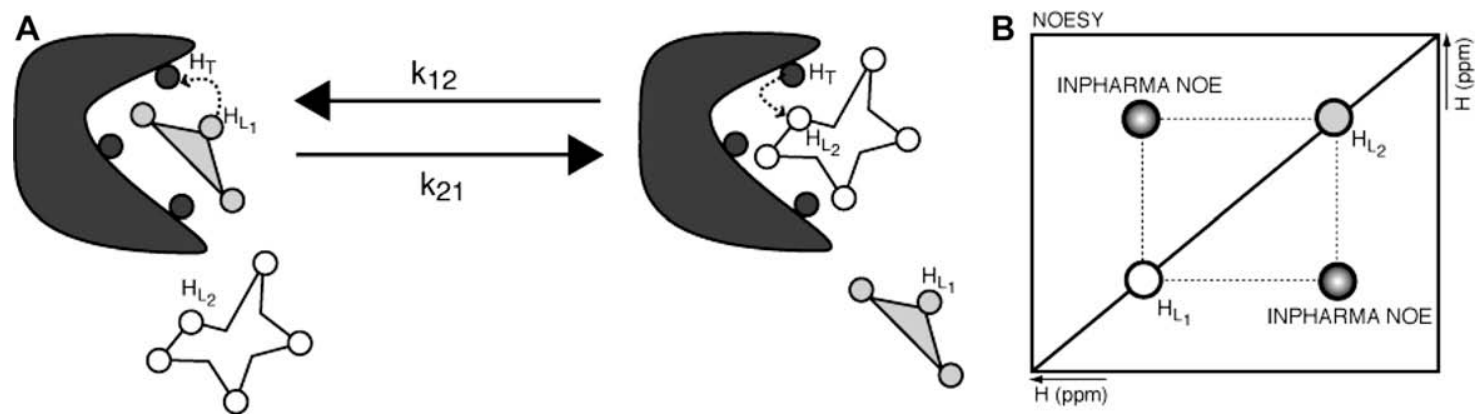

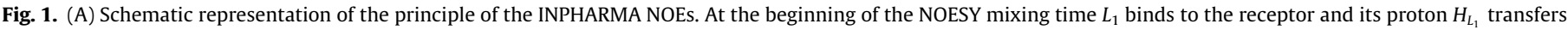

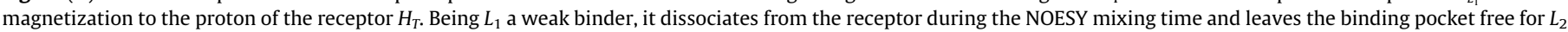

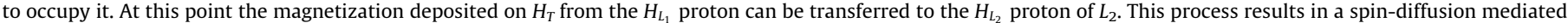
NOE peak between $H_{L_{1}}$ and $H_{L_{2}}$, depicted in (B).

magnetization transfer, the information gained in the INPHARMA spectrum is highly site-specific, in contrast to STD or WATERLOGSY experiments [8], which can only identify the face of the ligand that is solvent exposed with respect to that in contact with the receptor. The INPHARMA NOEs are used to rank and select binding modes of $L_{1}$ and $L_{2}$ obtained by docking the bound conformation of the ligands to a structural model of the apo-receptor. The direct employment of the INPHARMA NOEs in structure calculation programs (X-PLOR) is currently under development in our laboratories.

The method was originally developed to derive the binding mode of $L_{2}$ when the binding mode of $L_{1}$ is known. However, we have recently demonstrated that the INPHARMA approach is much more powerful and allows in favorable cases the de novo description of the binding mode of both $L_{1}$ and $L_{2}$ [11,12]. In this work we have shown that the methodology is precise enough to unambiguously select one docking mode per ligand and accurate enough to select the correct docking mode, as compared to the crystal structures available for the investigated test cases [12].

Here we present a thorough theoretical analysis of the INPHARMA interligand NOE effects. We use simple model systems to describe the effect of various parameters, such as receptor proton density, NOESY mixing time, ligand concentrations, etc., on the size of the observed INPHARMA NOEs. Furthermore, we present a comprehensive description of the applicability of the INPHARMA approach. We provide all tools necessary to predict the size of the INPHARMA NOEs expected for complexes with different $K_{d}$ and $k_{\text {off }}$ values and to choose the best conditions, in terms of sample composition and experimental NMR parameters, for the observation of INPHARMA NOEs of respectable size. The theoretical data presented here are essential to ensure that the powerful INPHARMA method can be widely applied by non-NMR experts in the process of drug development.

\section{Material and methods}

\subsection{The model systems}

The system that can be investigated with the INPHARMA method consists of a macromolecular receptor, usually a protein, and two competitively binding ligands. In this theoretical investigation we use simplified model systems to describe the influence of various structural parameters, such as intermolecular proton distances, proton densities in the receptor, depth of the binding pocket, etc., on the expected INPHARMA NOEs. Two simple geometries are used: (i) linear model: the ligands and the receptor consist of protons disposed in a linear arrangement with a fixed intra-molecular inter-proton distance $d$. The ligands contain from
1 to 3 protons (Fig. 2A); (ii) cubic model: the ligands consist of two or three protons linearly arranged with an inter-proton distance $d$; the receptor is a cube of different size $\left(5^{3}-9^{3}\right)$. In the receptor/ligand complex the protons are located at the nodes of a three-dimensional lattice with unit length $d$ in all three dimensions (Fig. 2B). In the free receptor, the nodes of the binding pocket are empty. The ligands insert perpendicular to one face, in the middle of it, so that the last atom is part of this face.

\subsection{Theory}

Intensities of NOE cross-peaks of a receptor/ligand complex in exchange with the free forms of both the ligand and the receptor are described by the following equation:

$\frac{d \mathbf{M}(t)}{d t}=-(\mathbf{R}+\mathbf{K}) \cdot\left(\mathbf{M}(t)-\mathbf{M}_{0}\right)$

with a solution of the form:

$\mathbf{M}\left(\tau_{m}\right)=\exp \left(-(\mathbf{R}+\mathbf{K}) \tau_{m}\right) \cdot\left(\mathbf{M}(0)-\mathbf{M}_{0}\right)+\mathbf{M}_{0}$
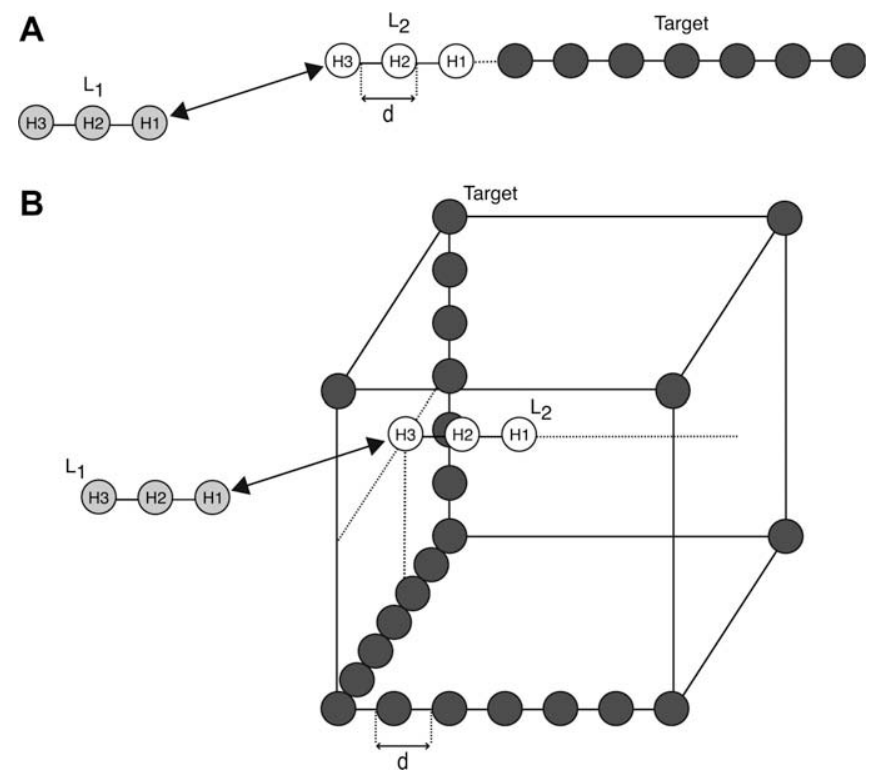

Fig. 2. Simplified models of the system consisting of one receptor (in black) and two competitively binding ligands (in gray and white). (A) Linear arrangement of the protons of both the receptor and the ligands; (B) linear arrangement for the protons of the ligands but cubic arrangement for the protons of the receptor. The ligands bind perpendicular to one face of the cube such that $\mathrm{H}_{3}$ is in the middle of this face. 
where $\mathbf{K}$ and $\mathbf{R}$ are the kinetic and relaxation matrices, $\mathbf{M}(0)$ the initial magnetization, $\mathbf{M}_{0}$ the equilibrium magnetization and $\tau_{m}$ the mixing time.

Two different models have been considered for the chemical exchange. The first is a three-step model, including the state where both ligands and the receptor are found also in the free form, as described by:

$T L_{1}+L_{2} \underset{k_{1 o n}}{\stackrel{k_{1} \text { off }}{\rightleftharpoons}} L_{1}+T+L_{2} \underset{k_{2 o f f}}{\stackrel{k_{2} \text { on }}{\rightleftharpoons}} L_{1}+T L_{2}$

In the second model, we assume that the receptor is never in the unbound state, due to presence of the two ligands in large excess. This model is described by:

$T L_{1}+L_{2} \underset{k_{21}}{\stackrel{k_{12}}{\rightleftharpoons}} L_{1}+T L_{2}$

\subsubsection{Model 1}

The relaxation matrix is a diagonal block matrix, where each submatrix describes the proton-proton relaxation pathway of one species; the species present in solution are the two ligands in the free state $L_{1}, L_{2}$, the two complexes $T L_{1}$ and $T L_{2}$ of the target macromolecule and $L_{1}$ or $L_{2}$, respectively [16], and the free target macromolecule $T$. The superindex of each $\mathbf{R}_{x}{ }^{s}$ indicates the species (either the free ligands and the receptor or the complexes $T L_{1}$ and $T L_{2}$ ) and the subindex indicates which protons of the species contribute to that sub-matrix. Thus, $\mathbf{R}_{L_{1}}^{L_{1}}$ describes the relaxation of the protons of ligand $L_{1}$ in the free state and $\mathbf{R}_{L_{1}}^{T L_{1}}$ the relaxation of the protons of ligand $L_{1}$ when bound in the complex $T L_{1}$. Analogously, $\mathbf{R}_{T}^{T L_{1}}$ describes the relaxation of the protein protons in the complex $T L_{1}$ and $\mathbf{R}_{L_{1}, T}^{T L_{1}}$ contains the cross-relaxation terms between the protons of ligand $L_{1}$ and the protons of protein $T$ in the complex $T L_{1}$.

$\mathbf{R}=\left(\begin{array}{ccccccc}R_{L_{1}}^{L_{1}} & & & & & & \\ & R_{L_{2}}^{L_{2}} & & & & & \\ & & R_{L_{1}}^{T L_{1}} & R_{L_{1}, T}^{T L_{1}} & & & \\ & & R_{T, L_{1}}^{T L_{1}} & R_{T}^{T L_{1}} & & & \\ & & & & R_{L_{2}}^{T L_{2}} & R_{L_{2}, T}^{T L_{2}} & \\ & & & & R_{T, L_{2}}^{T L_{2}} & R_{T}^{T L_{2}} & \\ & & & & & & R_{T}^{T}\end{array}\right)$

The kinetic matrix is built according to the equations that rule the chemical equilibrium:

$\frac{d\left[L_{1}\right]}{d t}=-k_{1 o n} \cdot[T]\left[L_{1}\right]+k_{1 o f f} \cdot\left[T L_{1}\right]$

$\frac{d\left[T L_{1}\right]}{d t}=-\frac{d\left[L_{1}\right]}{d t}$

$\frac{d\left[L_{2}\right]}{d t}=-k_{2 o n} \cdot[T]\left[L_{2}\right]+k_{2 o f f} \cdot\left[T L_{2}\right]$

$\frac{d\left[T L_{2}\right]}{d t}=-\frac{d\left[L_{2}\right]}{d t}$

$\frac{d[T]}{d t}=-\frac{d\left[T L_{1}\right]}{d t}-\frac{d\left[T L_{2}\right]}{d t}$

and has the form:

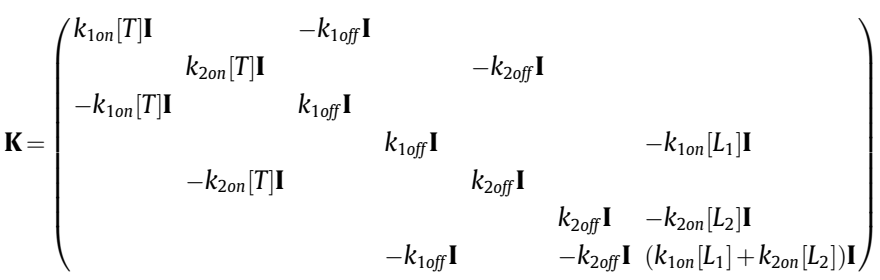

where $\mathbf{I}$ is the identity matrix of the same size as the corresponding block matrix in $\mathbf{R}$.

\subsubsection{Model 2}

Compared to the matrix for model 1 , the relaxation matrix $\mathbf{R}$ for model 2 lacks the last block representing the unbound target macromolecule.

$\mathbf{R}=\left(\begin{array}{cccccc}R_{L_{1}}^{L_{1}} & & & & & \\ & R_{L_{2}}^{L_{2}} & & & & \\ & & R_{L_{1}}^{T L_{1}} & R_{L_{1}, T}^{T L_{1}} & & \\ & & R_{T, L_{1}}^{T L_{1}} & R_{T}^{T L_{1}} & & \\ & & & & R_{L_{2}}^{T L_{2}} & R_{L_{2}, T}^{T L_{2}} \\ & & & & R_{T, L_{2}}^{T L_{2}} & R_{T}^{T L_{2}} \\ & & & & & \end{array}\right)$

The kinetics matrix $\mathbf{K}$ is derived from the equations:

$$
\begin{aligned}
& \frac{d\left[L_{1}\right]}{d t}=k_{12} \cdot\left[T L_{1}\right]\left[L_{2}\right]-k_{21} \cdot\left[T L_{2}\right]\left[L_{1}\right] \\
& \frac{d\left[T L_{1}\right]}{d t}=-\frac{d\left[L_{1}\right]}{d t} \\
& \frac{d\left[L_{2}\right]}{d t}=-\frac{d\left[L_{1}\right]}{d t} \\
& \frac{d\left[T L_{2}\right]}{d t}=\frac{d\left[L_{1}\right]}{d t} \\
& \left.\begin{array}{llllll}
k_{21}\left[T L_{2}\right] \mathbf{I} & & -k_{12}\left[L_{2}\right] \mathbf{I} & & (9) \\
-k_{21}\left[T L_{2}\right] \mathbf{I} & k_{12}\left[T L_{1}\right] \mathbf{I} & & -k_{21}\left[L_{1}\right] \mathbf{I} & \\
& & k_{12}\left[L_{2}\right] \mathbf{I} & & \\
& -k_{12}\left[T L_{1}\right] \mathbf{I} & k_{12}\left[L_{2}\right] \mathbf{I} & & -k_{21}\left[L_{1}\right] \mathbf{I} \\
& & & k_{21}\left[L_{1}\right] \mathbf{I} & \\
& & -k_{12}\left[L_{2}\right] \mathbf{I} & k_{21}\left[L_{1}\right] \mathbf{I}
\end{array}\right)
\end{aligned}
$$

where I is the identity matrix of the same size as the corresponding block matrix in $\mathbf{R}$.

The elements of the kinetics matrices of the two models are derived from the binding constants and the equilibrium concentrations. The last are calculated from the mass conservation laws.

Model 1:

$k_{2 o n}[T]\left[L_{2}\right]=k_{2 o f f}\left[T L_{2}\right]$

$k_{1 \text { on }}[T]\left[L_{1}\right]=k_{1 \text { off }}\left[T L_{1}\right]$

$\left[T L_{1}\right]+\left[T L_{2}\right]+[T]=[T]_{\text {tot }}$

$\left[T L_{1}\right]+\left[L_{1}\right]=\left[L_{1}\right]_{\text {tot }}$

$\left[T L_{2}\right]+\left[L_{2}\right]=\left[L_{2}\right]_{t o t}$

Model 2:

$k_{12}\left[T L_{1}\right]\left[L_{2}\right]=k_{21}\left[T L_{2}\right]\left[L_{2}\right]$

$\left[T L_{1}\right]+\left[T L_{2}\right]+[T]=[T]_{\text {tot }}$

$\left[T L_{1}\right]+\left[L_{1}\right]=\left[L_{1}\right]_{\text {tot }}$

$\left[T L_{2}\right]+\left[L_{2}\right]=\left[L_{2}\right]_{t o t}$

Individual elements of the relaxation matrix have the following form:

$R_{i, i}=\rho_{i}=\sum_{\substack{H_{j} \in A \\ j \neq i}} \frac{b^{2}}{d_{i j}^{6}} \cdot(J(0)+3 J(\omega)+6 J(2 \omega))$

$R_{i, j}=\sigma_{i j}=\frac{b^{2}}{d_{i j}^{6}} \cdot(6 J(2 \omega)-J(0))$ 
$J(\omega)=\frac{2}{5}\left(\frac{\tau_{c}}{1+\left(\omega \tau_{c}\right)^{2}}\right)$

$b=\frac{1}{2} \cdot \frac{\mu_{0}}{4 \pi} h \gamma_{H}^{2}$

where $\rho_{i}$ is the longitudinal relaxation rate of proton $H_{i}, \sigma_{i j}$ is the cross-relaxation rate of protons $H_{i}$ and $H_{j}, d_{i j}$ is the distance between protons $H_{i}$ and $H_{j}$ in the same chemical species (ligand, target macromolecule or complex) and "A" stand for the chemical species. All protons $H_{j}$ are considered in the calculation of the relaxation rates $\rho_{i}$ and $\sigma_{i j}$, irrespectively of the value of $d_{i j}$.

\subsection{Analytical solution for a three spins system}

A description of the transferred-NOE effect for a two-spin system has been given in great detail [5,17-19]. In these studies the receptor was not included in the calculation, namely it was assumed that it does not contribute considerably to the process of magnetization transfer between the ligand protons. However, it has been reported that the contribution of the receptor cannot be neglected for an accurate prediction of transferred-NOEs [16,20-24]. Clearly, for the calculation of the INPHARMA NOEs, the receptor protons must be taken into account as they mediate the transfer of magnetization between the two ligands. An analytical solution that describes the INPHARMA NOE transfer cannot be systematically found for a three spin system, where the two ligands $L_{1}$ and $L_{2}$ and the receptor $T$ consist of one proton each, as this would correspond to searching for an analytical solution of a polynomial equation of sixth order [25]. However, an analytical description of the INPHARMA transfer can be found by making two crude approximations: (1) the concentrations of the ligands and the receptor are equal; (2) all four species $L_{1}, L_{2}, T L_{1}$ and $T L_{2}$ have the same auto-relaxation rates $\rho$ and the cross-correlated relaxation rate $\sigma$ between the $H_{T}$ and $H_{L}$ protons in $T L_{1}$ and $T L_{2}$ are equal. Furthermore, the affinity of the two ligands is also chosen to be equal.

The first two approximations are clearly not realistic. However, an "easy-to-read" analytical solution for the three-spin system, even under the two coarse approximations made above, is still useful to describe at a glance the overall dependence of the INPHARMA magnetization transfer on internal dynamics and correlation time.

In the next section we show results calculated numerically for realistic scenarios with real physical constants and for multi-spin systems.

Under the approximations mentioned above, the relaxation matrix and the kinetics matrix simplify to the following form:

$$
\mathbf{R}+\mathbf{K}=\left(\begin{array}{cccccc}
k+\rho & 0 & -k & 0 & 0 & 0 \\
0 & k+\rho & 0 & 0 & -k & 0 \\
-k & 0 & k+\rho & \sigma & 0 & 0 \\
0 & 0 & \sigma & k+\rho & 0 & -k \\
0 & -k & 0 & 0 & k+\rho & \sigma \\
0 & 0 & 0 & -k & \sigma & k+\rho
\end{array}\right)
$$

where $k=k_{12}=k_{21}$. Solving Eq. (1) implies finding six eigenvalues that satisfy:

$\operatorname{det}(\mathbf{R}+\mathbf{K}-\lambda \mathbf{I})=0$

The INPHARMA NOE $S(t)$ between $L_{1}$ and $L_{2}$ is defined by:

$S(t)=-k^{3} \sigma^{2} \sum_{i=1}^{6} \alpha_{i} \cdot e^{-\lambda_{i} t}$

where $\alpha_{i}$ are coefficients that fulfilled the following equation system:

$$
\left(\begin{array}{cccccc}
1 & 1 & 1 & 1 & 1 & 1 \\
\lambda_{1} & \lambda_{2} & \lambda_{3} & \lambda_{4} & \lambda_{5} & \lambda_{6} \\
\lambda_{1}^{2} & \lambda_{2}^{2} & \lambda_{3}^{2} & \lambda_{4}^{2} & \lambda_{5}^{2} & \lambda_{6}^{2} \\
\lambda_{1}^{3} & \lambda_{2}^{3} & \lambda_{3}^{3} & \lambda_{4}^{3} & \lambda_{5}^{3} & \lambda_{6}^{3} \\
\lambda_{1}^{4} & \lambda_{2}^{4} & \lambda_{3}^{4} & \lambda_{4}^{4} & \lambda_{5}^{4} & \lambda_{6}^{4} \\
\lambda_{1}^{5} & \lambda_{2}^{5} & \lambda_{3}^{5} & \lambda_{4}^{5} & \lambda_{5}^{5} & \lambda_{6}^{5}
\end{array}\right) \cdot\left(\begin{array}{l}
\alpha_{1} \\
\alpha_{2} \\
\alpha_{3} \\
\alpha_{4} \\
\alpha_{5} \\
\alpha_{6}
\end{array}\right)=\left(\begin{array}{l}
0 \\
0 \\
0 \\
0 \\
0 \\
1
\end{array}\right)
$$

as described in Ref. [26]. The solution can be calculated from the known determinant of the Vandermonde matrix of Eq. (17):

$$
\begin{aligned}
& S(t)=-k^{3} \sigma^{2} \sum_{i-1}^{6} \Pi\left(\lambda_{i}\right) \cdot e^{-\lambda_{i} t} \\
& \Pi\left(\lambda_{i}\right)=\prod_{\substack{j=1 \\
j \neq i}}^{6}\left(\lambda_{i}-\lambda_{j}\right)^{-1}=\alpha_{i} \\
& \lambda_{i=1,3,5}=\frac{1}{3}(4 k+3 p)-\frac{2}{3} \sqrt{4 k^{2}+3 \sigma^{2}} \cos \theta_{i} \\
& \lambda_{i=2,4,6}=\frac{1}{3}(2 k+3 p)-\frac{2}{3} \sqrt{4 k^{2}+3 \sigma^{2}} \cos \theta_{i} \\
& \theta_{i}=\frac{1}{3}\left\{\tan ^{-1}\left(\frac{3 \sqrt{3\left(32 k^{4} \sigma^{2}+19 k^{2} \sigma^{4}+4 \sigma^{6}\right)}}{-16 k^{3}+9 k \sigma^{2}}\right)\right. \\
& \left.+\frac{\pi}{2}\left(1-\operatorname{Sign}\left(-16 k^{3}+9 k \sigma^{2}\right)\right)-i \pi\right\}
\end{aligned}
$$

Even with the drastic approximations made for this model system, the solution turns out to be quite complicated. However, a straightforward formula is obtained for the asymptotic development of the variable $k$ towards very high values (Fig. 3 ) and lead to the canonical expression of the INPHARMA NOE:

$S(t) \underset{k \rightarrow \infty}{\sim} \frac{1}{4} \cdot e^{-\rho t}\left(\cosh \left(\frac{\sigma t}{\sqrt{2}}\right)-1\right)$

This expression is reminiscent of the well known dependence of transferred-NOEs intensities on the mixing time $t$ of the NOESY experiment:

$S(t) \underset{k \rightarrow \infty}{\sim} \frac{1}{4} \cdot e^{-\rho t} \cdot \sinh \left(\frac{\sigma t}{\sqrt{2}}\right)$

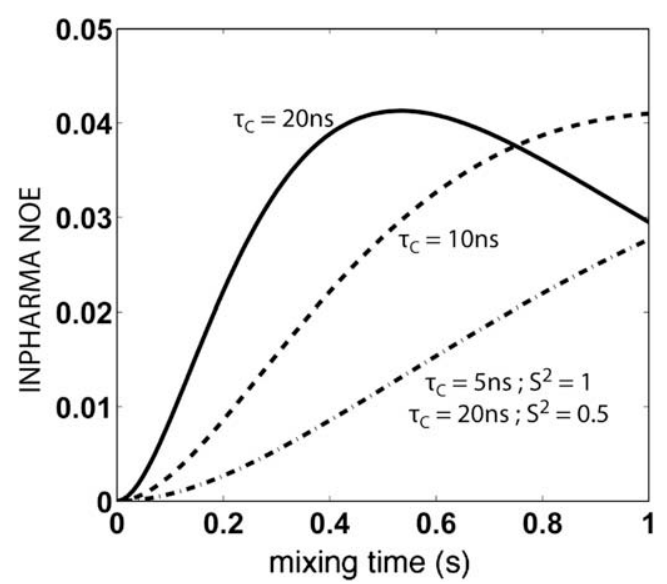

Fig. 3. Theoretical dependence of the INPHARMA NOEs on the mixing time $\tau_{m}$ under the approximations used to derive the analytical expression of Eqs. (19) and (21). The inter-proton distances $d$ are equal to $2.5 \AA$. The full line curve was calculated assuming $\tau_{c}=20 \mathrm{~ns}$ and $S^{2}=1$. The dashed line curve was calculated assuming $\tau_{c}=10 \mathrm{~ns}$ and $S^{2}=1$. The dotted-dashed line curve was calculated assuming $\tau_{c}=5 \mathrm{~ns}$ and $S^{2}=1$. The last curve was calculated assuming $\tau_{c}=20 \mathrm{~ns}$ and $S^{2}=0.5$ (overlapped with the dotted-dashed curve). Other parameters are field strength $=800 \mathrm{MHz},[T]_{\text {tot }}=50 \mu \mathrm{M},\left[L_{1}\right]_{\text {tot }}=\left[L_{2}\right]_{\text {tot }}=500 \mu \mathrm{M}, k_{\text {off }}=1 \mathrm{kHz}$ 
However, while the transferred-NOEs intensities depend on the time $t$ according to the hyperbolic sine function, which can be approximated to a linear function at small mixing times, the INPHARMA NOEs show a hyperbolic cosine dependence on time, which can be approximated by a parabolic curve at short mixing times. This behavior reveals the spin-diffusion nature of the INPHARMA NOEs, which depend quadratically on $\sigma t$.

The formula in Eq. (19) can be used to visualize the influence of the correlation time and internal motions on the build-up of the INPHARMA NOE. The effect of internal motions is estimated using the Lipari-Szabo's model-free approach in the limit of a very fast internal correlation time.

$S(t) \underset{k \rightarrow \infty}{\sim} \frac{1}{4} \cdot e^{-S^{2} \rho t}\left(\cosh \left(\frac{S^{2} \sigma t}{\sqrt{2}}\right)-1\right)$

where $S$ is the order parameter varying from 0 to 1 and assuming equal order parameters for the auto-relaxation rate $\rho$ and the cross-relaxation rate $\sigma$.

In Fig. 3, we show the dependence of the INPHARMA NOEs on the mixing time $t$, according to Eq. (21), for different correlation times of the molecule $\tau_{c}$ and different order parameters $S$. At short mixing times the curves have a parabolic shape, according to:

$S(t) \underset{\substack{k \rightarrow \infty \\ t \rightarrow 0}}{\sim} \frac{1}{4} \cdot\left(\frac{S^{2} \sigma t}{\sqrt{2}}\right)^{2}$

For $S^{2} \leqslant 1$, the cross-relaxation rate $\sigma$ is slower resulting in a delayed build-up of the INPHARMA NOEs. As for the transferred-NOEs, the presence of an order parameter $S \neq 1$ has a differently strong effect on the INPHARMA NOE depending on the mixing time. At $1 \mathrm{~s}$ mixing time the difference is negligible while at $400 \mathrm{~ms}$, there is a factor of four between the INPHARMA NOE assuming $S^{2}=1$ and $S^{2}=0.5$ (full line and dashed-dotted line in Fig. 3). This difference has to be taken in account in the interpretation of the INPHARMA NOE peaks that are transferred by highly dynamic parts of the binding pocket. Efforts to include order parameters in the interpretation of the data are on-going in our laboratories.

\subsection{Numerical solution for a multi-spin system}

For more than three spins and for systems with realistic parameters, the equations system becomes too complex to search for an analytical solution. Therefore a numerical solution is calculated in Matlab for the linear and cubic model systems assuming both model
1 and model 2 for the chemical exchange and in dependence of physical parameters such as $\tau_{c}, \tau_{m}, k_{o f f}$, species concentrations and internal dynamics. The results of such simulations, which are essential to understand the influence of the physical and experimental parameters on the size of the observed INPHARMA NOEs, are presented in the following section.

\section{Results and discussion}

\subsection{Choice of the kinetic model}

One of the challenges of the INPHARMA method is to handle a large number of docking models for each of the two ligands, which need to be ranked with respect to the measured interligand NOEs. Thus, the calculation time needed to predict the interligand NOEs for one pair of models should be kept to a minimum. A medium size protein $(20 \mathrm{kDa})$ contains about 2000 protons which translates into a matrix $\mathbf{R}+\mathbf{K}$ of ca. twelve million elements. The size of the $\mathbf{R}+\mathbf{K}$ matrix can be reduced to eight millions by choosing the kinetic model 2, where the presence of the free receptor in solution is neglected. In order to evaluate the error introduced on the calculated INPHARMA NOEs by neglecting the presence of the free receptor in solution, we simulated the interligand NOEs between the two protons $H_{1}$ of $L_{1}$ and $H_{1}$ of $L_{2}$ in presence of a common receptor consisting of a cube of $7^{3}$ protons. In all simulations shown in this and the following paragraphs, the magnetization originates from $L_{1}$ and is transferred to $L_{2}$; the INPHARMA NOE shown on the $y$ axis is normalized to the diagonal peak of the proton $H_{1}$ of $L_{1}$ in a NOESY experiment with mixing time $\tau_{m}=0$; a diffusion limited $k_{\text {on }}$ of $10^{8} \mathrm{M}^{-1} \mathrm{~s}^{-1}$ is assumed; for the cubic model system (Fig. 2B) the ligand is placed deep in the receptor cube with the ligand proton $H_{1}$ at coordinates $(4,4,3)$, unless stated otherwise. Clearly the error introduced by using the approximated model 2 depends on the amount of receptor free in solution at equilibrium. For a system consisting of $\left[L_{1}\right]_{\text {tot }}=\left[L_{2}\right]_{\text {tot }}=500 \mu \mathrm{M}$, $[T]_{\text {tot }}=50 \mu \mathrm{M}$ and with $K_{d}=100 \mu \mathrm{M}$ for both ligands, the error made by neglecting the presence of the free receptor $(4 \mu \mathrm{M})$ varies with the mixing time of the NOESY experiment and reaches ca. $10 \%$ or $5 \%$ at $\tau_{m}=0.5 \mathrm{~s}$ or $1 \mathrm{~s}$, respectively (Fig. $4 \mathrm{~A}$ ). However, for tighter binding ligands with $K_{d}=10 \mu \mathrm{M}$ or lower, the presence of the free receptor $(0.5 \mu \mathrm{M})$ can be safely neglected, as the error on the interligand NOE is less than $3-5 \%$ over the complete range of mixing times from $0 \mathrm{~s}$ to $1 \mathrm{~s}$ (Fig. 4B). In our experience errors smaller than $5-10 \%$ are not expected to influence the outcome of the analysis.
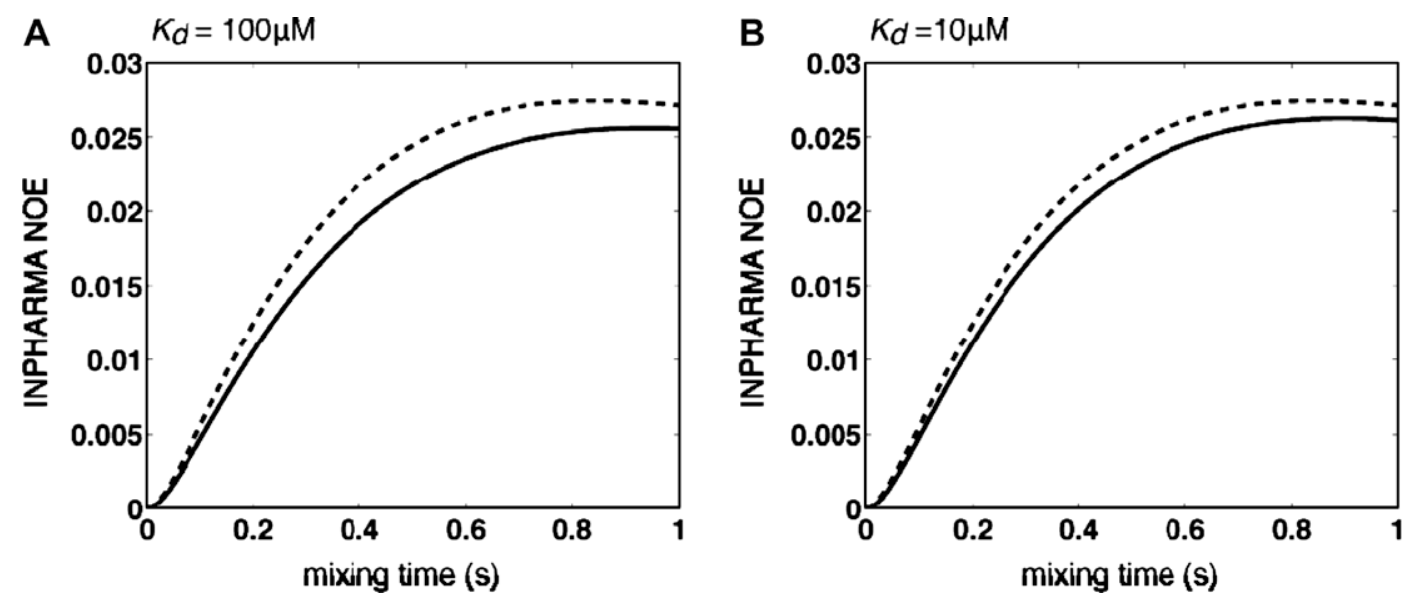

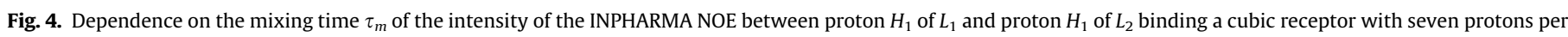

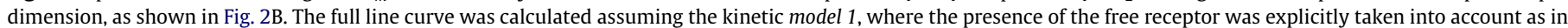

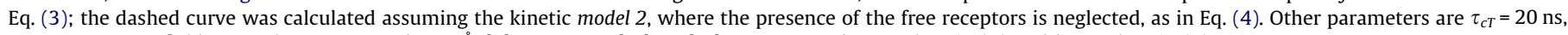
$\tau_{c A}=\tau_{c B}=0.1 \mathrm{~ns}$, field strength $=800 \mathrm{MHz}, d=2.5 \AA,[T]_{\text {tot }}=50 \mu \mathrm{M},\left[L_{1}\right]_{\text {tot }}=\left[L_{2}\right]_{\text {tot }}=500 \mu \mathrm{M} . k_{\text {off }}=10 \mathrm{kHz}$ in $(\mathrm{A})$ and $k_{\text {off }}=1 \mathrm{kHz}$ in $(\mathrm{B})$. 


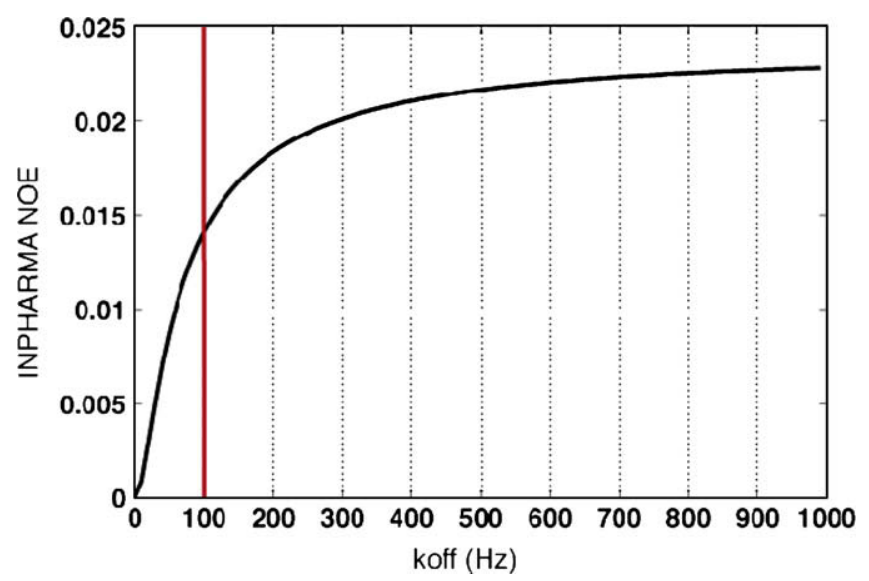

Fig. 5. Dependence on the $k_{\text {off }}=k_{1 \text { off }}=k_{2 \text { off }}$ of the intensity of the INPHARMA NOE between proton $H_{1}$ of $L_{1}$ and proton $H_{1}$ of $L_{2}$ binding a cubic receptor with seven protons per dimension, as shown in Fig. 2B. The curve was calculated assuming the kinetic model 1. Other parameters are $\tau_{m}=500 \mathrm{~ms}, \tau_{c T}=20 \mathrm{~ns}, \tau_{c A}=\tau_{c B}=0.1 \mathrm{~ns}$, field strength $=800 \mathrm{MHz}, d=2.5 \AA,[T]_{\text {tot }}=50 \mu \mathrm{M},\left[L_{1}\right]_{\text {tot }}=\left[L_{2}\right]_{\text {tot }}=500 \mu \mathrm{M}$.

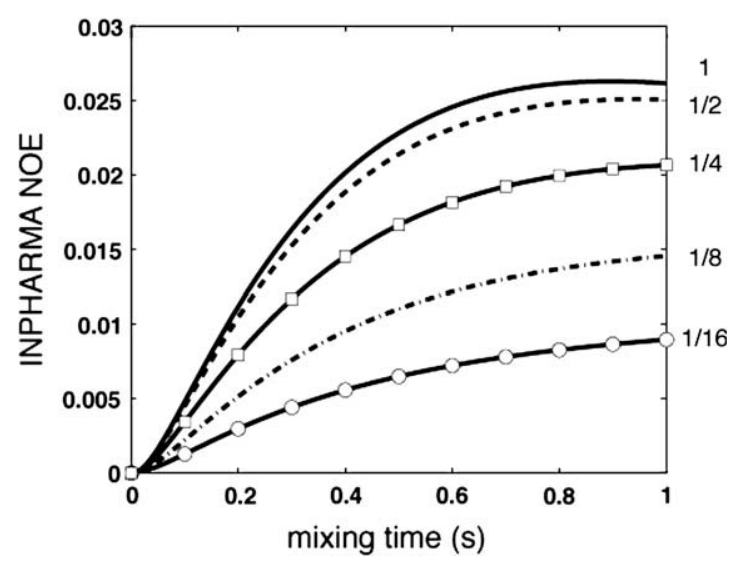

Fig. 6. Dependence on the mixing time $\tau_{m}$ of the intensity of the INPHARMA NOE between proton $H_{1}$ of $L_{1}$ and proton $H_{1}$ of $L_{2}$ binding a cubic receptor with seven protons per dimension, for different $k_{1 \text { off }} / k_{2 \text { off }}$ ratios. The curves were calculated assuming the kinetic model 1 and $k_{2 o f f}=1 \mathrm{kHz}$. Other parameters are as in Fig. 5.

\subsection{Dependence on the $k_{\text {off }}$ of the two ligands}

In order to observe the INPHARMA NOEs, the two ligands should bind weakly to the receptor, namely they should exchange a few times between their free and bound states during the mixing time of a NOESY experiment. Fig. 5 shows the dependence on the $k_{\text {off }}$ of the INPHARMA NOE occurring between the two protons $H_{1}$ of $L_{1}$ and $H_{1}$ of $L_{2}$ in presence of a common receptor consisting of a cube of $7^{3}$ protons, assuming model 1 for the exchange and equal affinity for the two ligands. For a mixing time of $500 \mathrm{~ms}$ and a $k_{\text {off }}$ of $1 \mathrm{kHz}$ or higher (corresponding to a $K_{d}$ of $10 \mu \mathrm{M}$ if one assumes a diffusion limited $k_{o n}$ of $10^{8} \mathrm{M}^{-1} \mathrm{~s}^{-1}$ ) the INPHARMA NOE has reached its maximum value, while for a $k_{\text {off }}$ of $100 \mathrm{~Hz}$ the INPHARMA NOE has an observable size equal to about two third of its maximum value. These results indicate that the transfer of magnetization between the two ligands improves with the number of exchange events in the binding pocket of the receptor. The condition on the $k_{\text {off }}$ is not too stringent and the INPHARMA NOE could be observed for most weakly binding ligands, typically available in an early phase of drug development, which we tested.

More critical is the relative size of the dissociation rates for $L_{1}$ and $L_{2}, k_{1 o f f}$ and $k_{2 o f f}$. Clearly, the optimal situation for the receptor mediated transfer of magnetization between the two ligands is when $k_{1 \text { off }}=k_{2 \text { off }}$ and the two ligands spend an equal amount of time in the receptor bound state. Fig. 6 shows the efficiency of the INPHARMA NOE transfer when $k_{1 \text { off }} / k_{2 \text { off }} \neq 1$. A considerable amount of magnetization transfer through the INPHARMA NOE is obtained for a $k_{1 \text { off }} / k_{2 \text { off }}$ value of up to 8 , while for $k_{1 \text { off }} / k_{2 \text { off }}=16$ the INPHARMA NOE is less than $1 \%$ at a mixing time of $1 \mathrm{~s}$ and has decreased by a factor of 4 at $500 \mathrm{~ms}$ with respect to $k_{1 \text { off }}=k_{2 o f f}$. Thus, the method requires that the affinity of the two ligands is of comparable size.

An obvious way to compensate for the different affinity of the two ligands, and consequently for the different equilibrium concentrations of the $T L_{1}$ and $T L_{2}$ complexes in solution, might seem that of adjusting the concentration of the two ligands, as for example using $\left[L_{1}\right]_{\text {tot }}=10\left[L_{2}\right]_{\text {tot }}$ if $k_{1 \text { off }}=10 k_{2 \text { off. }}$. At small mixing times, for which the two-step transfer dominates the INPHARMA signal, a concentration ratio $\left[L_{1}\right]_{\text {tot }}=10\left[L_{2}\right]_{\text {tot }}$ is optimal (Fig. 7). However, at longer mixing times the optimal concentration changes due to the contribution of additional pathways of magnetization transfer and spin-diffusion inside the receptor. The use of longer mixing times renders the analysis of the INPHARMA NOE intensity more
A

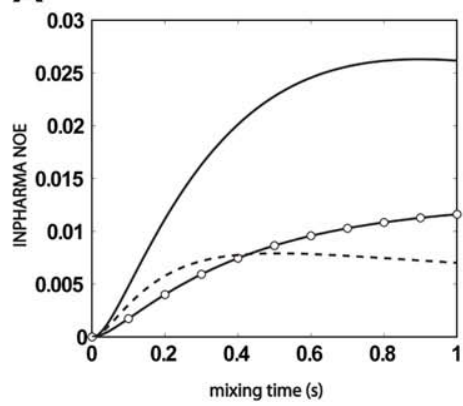

B

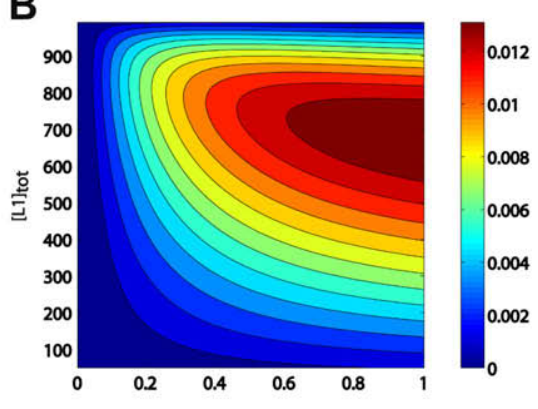

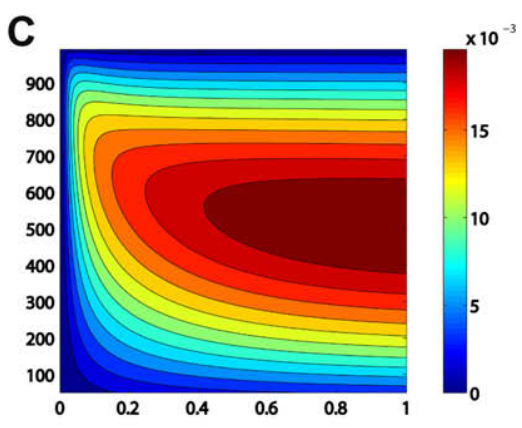

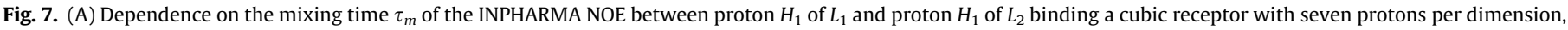

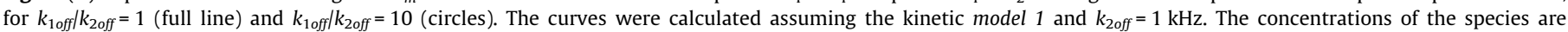

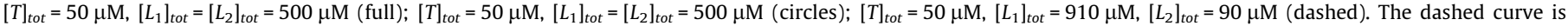

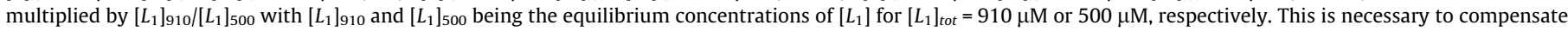

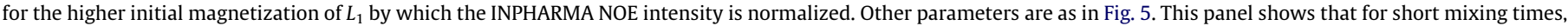

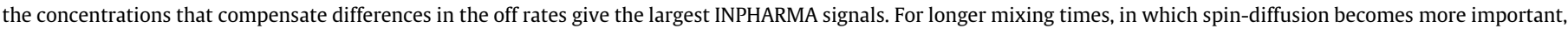

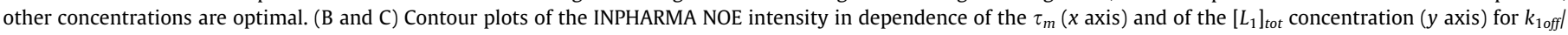

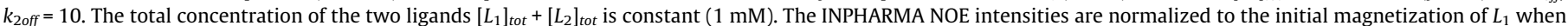

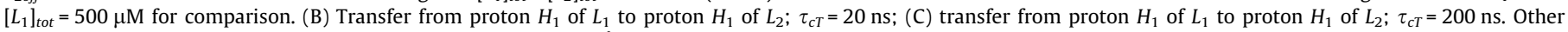
parameters are $\tau_{c A}=\tau_{c B}=0.1 \mathrm{~ns}$, field strength $=800 \mathrm{MHz}, d=2.5 \AA,[T]_{\text {tot }}=50 \mu \mathrm{M}$. 
difficult, due to the contribution of many spin-diffusion pathways; on the other hand long mixing times are necessary to increase the intensity of the INPHARMA signal.

For a complex with $\tau_{c}=20$ ns the optimal value is found around $\left[L_{2}\right]_{\text {tot }} /\left[L_{1}\right]_{\text {tot }}=0.33$ (Fig. 7B). On the other hand for a complex with $\tau_{c}=200 \mathrm{~ns}$ (Fig. 7C) the optimal INPHARMA NOE is found at long mixing times for similar concentrations of $L_{1}$ and $L_{2}$ ([ $\left.L_{2}\right]_{\text {tot }} /$ $\left.\left[L_{1}\right]_{\text {tot }}=0.81\right)$ despite the differences in the $k_{\text {off. }}$. In this case it is more important to achieve fast transfer of magnetization between the two ligands away from the fast relaxing receptor, in order to avoid the diffusion of the ligands magnetization in the receptor, rather than to have similar population of the $T L_{1}$ and $T L_{2}$ complexes.

Fig. 7A summarizes these results. Here the INPHARMA NOE in dependence of the mixing time for ligands with equal affinity and equal concentration (full line), as compared to ligands with $k_{1 \text { off }}=10 k_{2 \text { off }}$ (dashed line) is shown. Not unexpectedly, the optimal INPHARMA integrals are observed when the affinities and the concentrations of the two ligands are equal. Changing the ratio of $\left[L_{2}\right]_{\text {tot }} /\left[L_{1}\right]_{\text {tot }}$ from 1 to 0.1 (circles) while keeping the overall concentration of the ligands constant $\left(\left[L_{2}\right]_{\text {tot }}+\left[L_{1}\right]_{\text {tot }}=1 \mathrm{mM}\right)$ cannot compensate the decrease of the transfer efficiency due to unequal affinities. This result can be understood considering that while the $k_{\text {off }}$ represents the inverse of the lifetime of the complex, the concentration of the ligand influences the probability that the complex is formed. Thus, owing to the spin-diffusion loss between two consecutive complex formations, binding of the ligand to the receptor for twice as long does not have the same effect as binding twice as often. At shorter mixing times the INPHARMA NOE for $k_{1 \text { off }}=10 k_{2 o f f}$ and $\left[L_{2}\right]_{\text {tot }} /\left[L_{1}\right]_{\text {tot }}=0.1$ (dashed) is larger than for $k_{1 o f f}=10 k_{2 o f f}$ and $\left[L_{2}\right]_{\text {tot }} /\left[L_{1}\right]_{\text {tot }}=1$ (circles), while at longer mixing times the INPHARMA NOE shows the opposite behavior. The critical point where the INPHARMA NOE for $\left[L_{2}\right]_{\text {tot }} /\left[L_{1}\right]_{\text {tot }}=0.1$ becomes worse than for $\left[L_{2}\right]_{\text {tot }} /$ $\left[L_{1}\right]_{\text {tot }}=1$ depends on the correlation time of the complex and shifts to shorter mixing times for larger $\tau_{c}$.

\subsection{Influence of proton density}

An important question is how the number of protons of both the ligands and the receptor influences the INPHARMA NOEs. Clearly, the most efficient transfer is obtained when the magnetization is not allowed to diffuse away through undesired spin-diffusion pathways and only the desired spin-diffusion transfer $L_{1} \rightarrow$ receptor $\rightarrow L_{2}$ occurs. In a real system, however, this is never the case, due to the presence of protons in the ligands and in the receptor that do not belong to the binding epitope or to the binding pocket.

We first investigate the effect of the ligand proton density by comparing the INPHARMA NOE in a linear system, as in Fig. 2A, for the case when the two ligands consist of only one, two or three protons (Fig. 8). The presence of protons $H_{2}$ of $L_{1}$ and $L_{2}$ diminishes the efficiency of the transfer between $H_{1}$ of $L_{1}$ and $H_{1}$ of $L_{2}$ by a factor of three (Fig. 8B), due to the fact that the ligand protons $\mathrm{H}_{2}$ compete with the receptor protons for the $\mathrm{H}_{1}$ magnetization. However, the presence of additional ligand protons at $d \geqslant 5 \AA\left(H_{3}\right.$ of $L_{1}$ and $L_{2}$ ) does not further affect the efficiency of the INPHARMA NOE.

Due to the dependence of spin-diffusion on the size of the molecule, proton density on the large receptor is likely to have a stronger effect on the intensity of the INPHARMA NOE. To simulate the dependence of the INPHARMA NOE on the number of protons of the receptor, while keeping the $\tau_{c T}$ constant, we used the artificial cubic system of Fig. 2B. Fig. 9 shows the results of such simulations for a cube of size ranging from $5^{3}$ to $9^{3}$. As expected, the efficiency of magnetization transfer diminishes upon increasing the number of receptor protons, due to the undesired diffusion of the ligand(s) magnetization from the binding pocket to the receptor body. Such a loss reaches a maximum for a sphere of about $8 \AA$ around the ligand binding pocket (size of the cube $=7^{3}$ ); spin-diffusion processes beyond this sphere do not influence the INPHARMA NOE to a measurable extent for molecules with a $\tau_{c}$ similar to that used in the simulation (20 ns). Clearly, increasing the size of the receptor directly translates into a higher velocity of the spin-diffusion and to a larger radius of the sphere within which protons contribute to the magnetization transfer process. However, also for very large receptors the mixing time of the NOESY experiment can be optimized to obtain the maximum INPHARMA NOE transfer, while minimizing dilution of the signal throughout the receptor (see next paragraph). For a receptor with $\tau_{c}=20 \mathrm{~ns}$, we can conclude that spin-diffusion inside the receptor competes with the INPHARMA magnetization transfer up to a distance of $8 \AA$ from the binding pocket and can therefore be considered as a "local" process.

\subsection{Optimal choice of $\tau_{m}$ : dependence on $\tau_{c}$}

The mixing time that maximizes the intensity of the INPHARMA NOEs depends on the correlation time of the complex. Fig. 10 shows the dependence of the INPHARMA NOE on the mixing time $\tau_{m}$ for receptors with increasing $\tau_{c}$. At short mixing times the
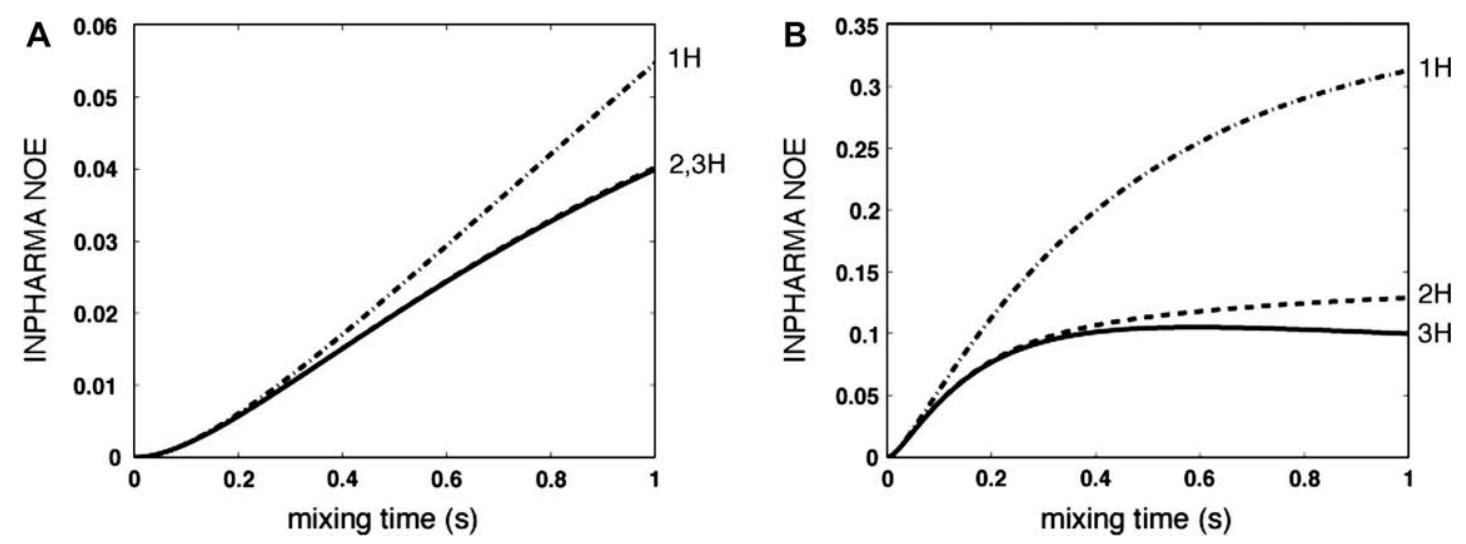

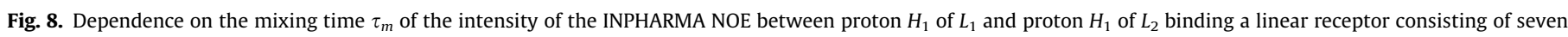

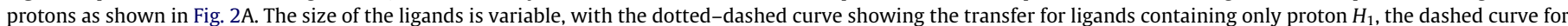

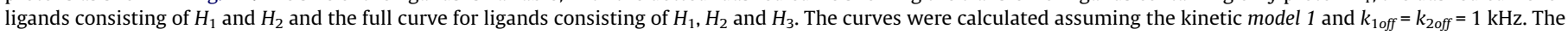
concentrations of the species are $[T]_{\text {tot }}=50 \mu \mathrm{M},\left[L_{1}\right]_{\text {tot }}=\left[L_{2}\right]_{\text {tot }}=500 \mu \mathrm{M}$. Curves in $(\mathrm{A})$ and (B) were calculated assuming $\tau_{c T}=20 \mathrm{~ns}$ and $\tau_{c T}=200 \mathrm{~ns}$, respectively. 


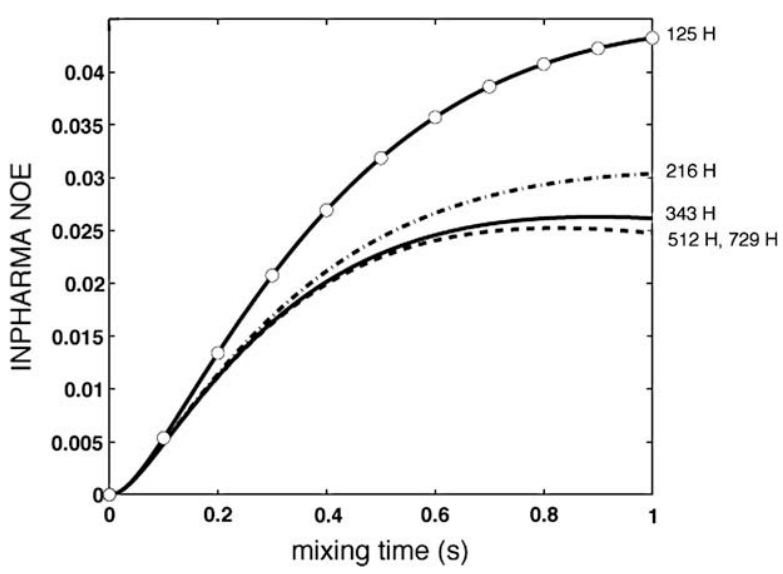

Fig. 9. Dependence on the mixing time $\tau_{m}$ of the intensity of the INPHARMA NOE between proton $H_{1}$ of $L_{1}$ and proton $H_{1}$ of $L_{2}$ binding a cubic receptor of variable size: $5^{3}$ (circles), $6^{3}$ (dotted-dashed), $7^{3}$ (full), $8^{3}$ and $9^{3}$ (dashed, overlapped). The curves were calculated assuming the kinetic model 1 . Other parameters are $\tau_{c T}=20 \mathrm{~ns}, \tau_{c A}=\tau_{c B}=0.1 \mathrm{~ns}$, field strength $=800 \mathrm{MHz}, d=2.5 \AA,[T]_{t o t}=50 \mu \mathrm{M}$, $\left[L_{1}\right]_{\text {tot }}=\left[L_{2}\right]_{\text {tot }}=500 \mu \mathrm{M}$.

INPHARMA NOE shows a parabolic dependence on $\tau_{m}$, which is typical of a second order effect (spin-diffusion) (see also Eq. (21)). Increasing the correlation time increases the steepness of the initial part of the curve.

As it is seen in Fig. 10B, for a receptor of $400 \mathrm{kDa}$ or larger, the maximum INPHARMA NOE is obtained for $\tau_{m}<100 \mathrm{~ms}$, while for a receptor of ca. $20 \mathrm{kDa}$, mixing times of the order of $500 \mathrm{~ms}$ or higher can be used. In our experience the best results, in terms of selection of the correct docking mode by comparison of the theoretical and experimental INPHARMA NOEs, are obtained when the experimental data are acquired at mixing times for which the INPHARMA NOE has not yet reached its maximum value. This is due to the fact that in this regime only "short" spin-diffusion pathways contribute to the signal, thus minimizing the errors made by neglecting internal motions in the calculation of the theoretical NOEs (vide infra). In fact, the more receptor protons contribute to the spin-diffusion transferred-NOEs, the largest is the error made by neglecting their internal dynamics.

\subsection{Dependence on receptor concentration}

Clearly the size of the INPHARMA NOE depends not only on the $\tau_{c}$ of the receptor, the affinity of the ligands to the receptor and the

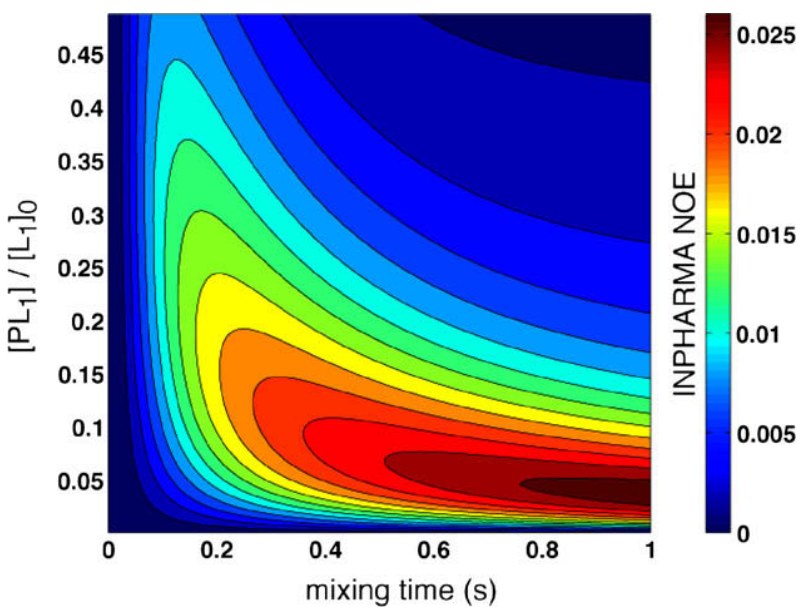

Fig. 11. Contour plot of the dependence of the INPHARMA NOE between proton $H_{1}$ of $L_{1}$ and proton $H_{1}$ of $L_{2}$ binding a cubic receptor of size $7^{3}$ on the $\tau_{m}(x$ axis) and on the fraction of bound ligand $\left[T L_{1}\right] /\left[L_{1}\right]_{0}$ ( $y$ axis). The curves were calculated assuming the kinetic model 1 . Other parameters are $\tau_{c T}=20 \mathrm{~ns}, \tau_{c A}=\tau_{c B}=0.1 \mathrm{~ns}$, field strength $=800 \mathrm{MHz}, d=2.5 \AA,\left[L_{1}\right]_{\text {tot }}=\left[L_{2}\right]_{\text {tot }}=500 \mu \mathrm{M}$.

kinetic constants but also on the concentration of the species in solution. In Fig. 11 we show a contour plot of the intensity of the INPHARMA NOE in dependence of the mixing time ( $x$ axis) and the fraction of bound ligand ( $y$ axis), defined as $\left[T L_{1}\right] /$ $\left[L_{1}\right]_{\text {tot }}=\left[T L_{2}\right] /\left[L_{2}\right]_{\text {tot }}$. The ligands total concentration is $500 \mu \mathrm{M}$ each. At short mixing times the INPHARMA NOE intensity linearly increases with the fraction of bound ligand. On the other hand, at long mixing times the intensity of the INPHARMA NOE steeply depends on the fraction of bound ligand. For $\tau_{m}>600 \mathrm{~ms}$ the optimal value of the INPHARMA NOE is achieved for a fraction of bound ligand ranging from 0.02 to 0.06 , whereas at $150 \mathrm{~ms}$ bound ligand fractions ranging from 0.1 to 0.35 are required for optimal signal. However, the best results can be obtained at long mixing times using a low fraction of bound ligand (Fig. 11).

\subsection{Effect of internal dynamics}

Dynamics plays an important role in the NOE transfer mechanisms. Campbell and Sykes evaluated the influence of internal motions of the bound and free ligand on the transferred-NOE effect neglecting the receptor protons [27]. Here, we analyze the effect of internal motions on the interligand INPHARMA NOE effect using the model-free approach of Lipari and Szabo [28].
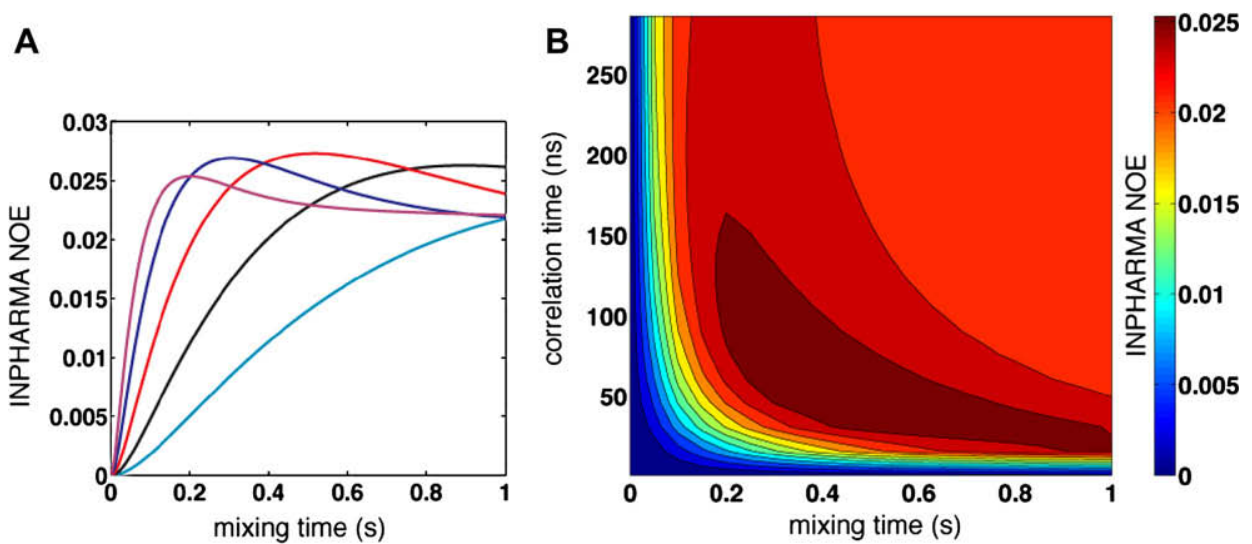

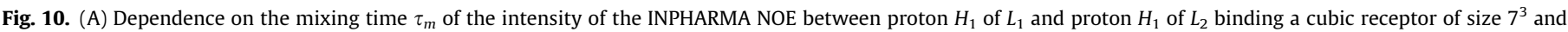

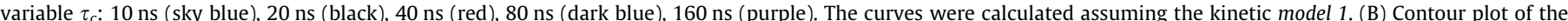

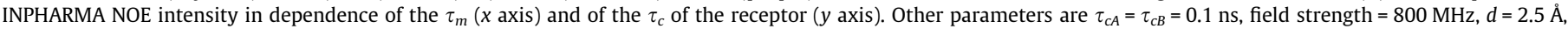
$[T]_{\text {tot }}=50 \mu \mathrm{M},\left[L_{1}\right]_{\text {tot }}=\left[L_{2}\right]_{\text {tot }}=500 \mu \mathrm{M}$. 


\section{A}

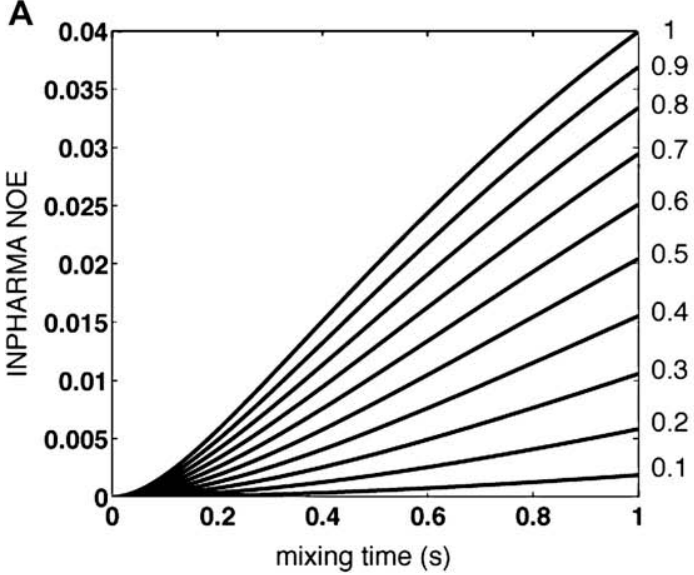

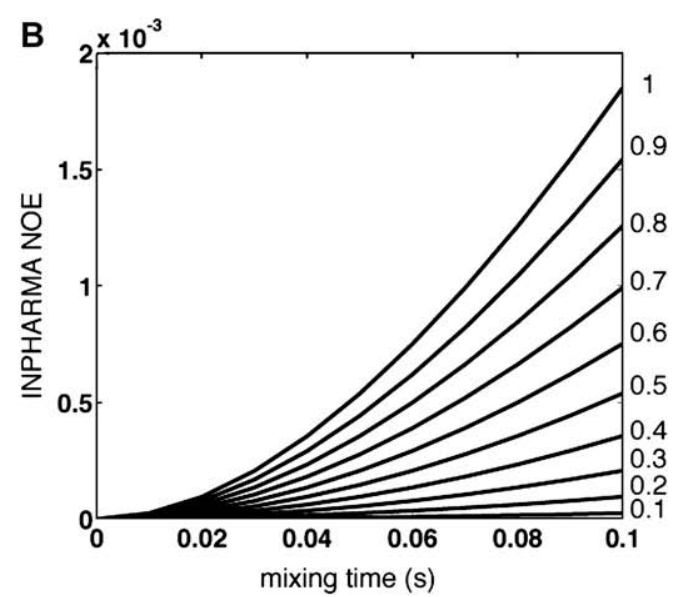

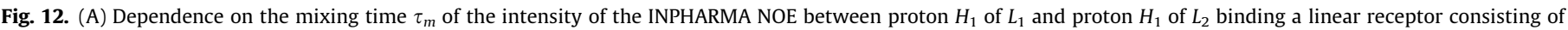

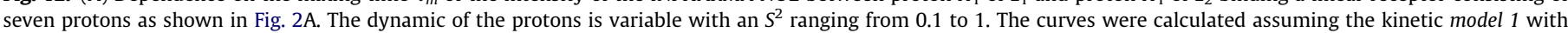

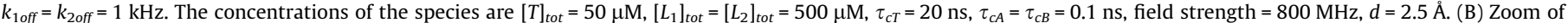
(A) for the mixing times between $0 \mathrm{~s}$ to $0.1 \mathrm{~s}$.

Clearly in this analysis we need to consider the receptor protons as well, as they mediate the transfer of magnetization between the two ligands.

In order to quantitatively evaluate the error made by neglecting internal dynamics in the back-calculation of the INPHARMA NOE from a certain docking model, we performed theoretical simulations of the INPHARMA NOE, using the cubic system of Fig. 2B, in presence of internal dynamics of variable amplitude. First we assumed the same order parameter $S^{2}$ for all protons in all species, both free and bound. Fig. 12 shows that the efficiency of the INPHARMA transfer decreases in presence of internal dynamics. At low mixing times the decrease of the magnetization transfer efficiency is proportional to $S^{4}$, as it was found in the analytical solution of Eq. (21). Thus the effect of internal motions at low mixing times corresponds to decreasing the correlation time of the species by a factor $S^{4}$ (Fig. 12B).

The best choice of the mixing time depends not only on the $\tau_{c}$ of the complex but also on the presence of internal motions. The optimal compromise between optimizing the intensities of the INPHARMA NOEs and minimizing the error generating from neglecting internal motions is achieved, in our experience, by acquiring the experimental data at mixing times for which the INPHARMA NOEs have not reached their maximum value, yet.

In a more realistic system, different internal dynamics occurs for each species. Therefore, we simulated the efficiency of the INPHARMA magnetization transfer using three different order parameters $S_{L}^{2}, S_{T}^{2}$ and $S_{T L}^{2}$, for the free ligands protons, the free receptor protons and the receptor protons or ligand protons in the complexes, respectively (Fig. 13). Our simulations show that the INPHARMA magnetization transfer is not considerably affected either by the internal dynamics of the free ligands or by that of the free receptor (provided that the concentration of the free receptor is not too high). On the other hand, internal dynamics in the complexes measurably affects the efficiency of the INPHARMA magnetization transfer. For a medium size receptor, the efficiency of magnetization transfer at constant $\tau_{m}$ decreases upon increasing the amplitude of internal dynamics either for the receptor or the ligands protons in the complex, whereby the effect of receptor protons dynamics is smaller. The reason for this lies in the two counteracting effects of the internal dynamics of the receptor protons: on the one hand, receptor protons dynamics reduces the spin-diffusion rate necessary for the INPHARMA transfer, thus resulting in smaller INPHARMA NOEs; on the other hand it reduces the rate of

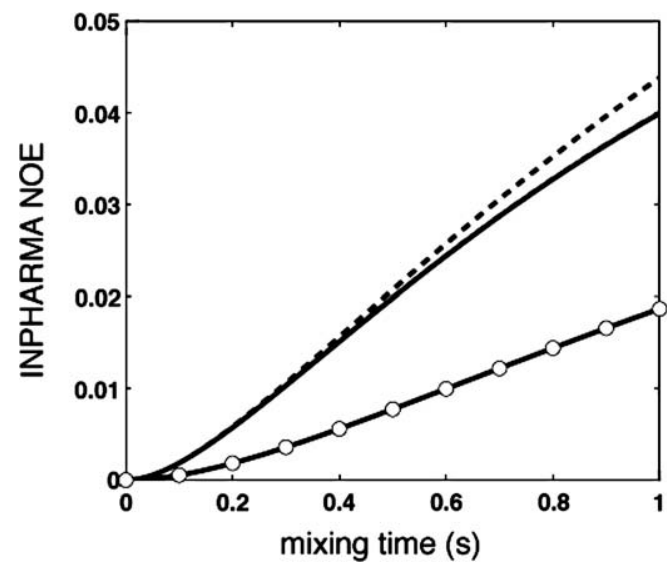

Fig. 13. Dependence on the mixing time $\tau_{m}$ of the intensity of the INPHARMA NOE between proton $H_{1}$ of $L_{1}$ and proton $H_{1}$ of $L_{2}$ binding a linear receptor consisting of seven protons as shown in Fig. 2A. Different amount of dynamics are taken into account: (1) no dynamics is present either for the ligands or for the receptor: $S^{2}=1$ (full line); (2) the free ligand is dynamic with an $S_{L}^{2}=0.5$ (dashed line); ( 3 ) the free receptor is dynamic with an $S_{T}^{2}=0.5$ (overlapped with the full line); (4) both the ligands and the receptor in the complex show dynamics with $S_{T L}^{2}=0.5$ (full line with circles). The curves were calculated assuming the kinetic model 1 with $k_{1 \text { off }}=k_{2 \text { off }}=1 \mathrm{kHz}$. The concentrations of the species are $[T]_{\text {tot }}=50 \mu \mathrm{M},\left[L_{1}\right]_{\text {tot }}=\left[L_{2}\right]-$ tot $=500 \mu \mathrm{M}, \tau_{c T}=20 \mathrm{~ns}, \tau_{c A}=\tau_{c B}=0.1 \mathrm{~ns}$, field strength $=800 \mathrm{MHz}, d=2.5 \AA$.

diffusion of the magnetization away from the binding pocket into the receptor body, thus improving the efficiency of the INPHARMA magnetization transfer.

\section{Conclusions}

The INPHARMA method is a flexible, rapid and powerful technique to determine the relative, and in some favorable cases even the absolute, orientations of two ligands binding weakly and competitively to the same binding pocket of a common receptor. This methodology is particularly useful in the process of optimizing drug leads as it provides a fast method to determine the relative orientation of different chemical skeletons in the receptor binding pocket.

Here we provide a theoretical description of the INPHARMA effect and we describe its dependence on both kinetic and experi- 
mental variables. The equations and the graphs shown here provide an arsenal of tools to determine the applicability of the method to specific cases and to correctly choose the experimental parameters. We show that the choice of the experimental parameters, as for example the $\tau_{m}$ of the NOESY experiments, is critical and strictly depends on the system under investigation. Furthermore, we provide an estimation of the errors committed by using approximations, as for example neglecting the effect of internal motions.

The analysis presented here helps to optimize the results from INPHARMA measurements.

\section{Acknowledgments}

This work was supported by the Fonds der Chemischen Industrie (fellowship to J.O. No.: 180081, support to C.G.), the MPG, and the EMBL. We thank Dr. D. Lee for discussion about the analytical solution of the INPHARMA NOE.

\section{References}

[1] T. Carlomagno, W. Bermel, C. Griesinger, Measuring the chi 1 torsion angle in protein by $\mathrm{CH}-\mathrm{CH}$ cross-correlated relaxation: a new resolution-optimised experiment, J. Biomol. NMR 27 (2003) 151-157.

[2] T. Carlomagno, M.J. Blommers, J. Meiler, B. Cuenoud, C. Griesinger, Determination of aliphatic side-chain conformation using cross-correlated relaxation: application to an extraordinarily stable 2 -aminoethoxy-modified oligonucleotide triplex, J. Am. Chem. Soc. 123 (2001) 7364-7370.

[3] T. Carlomagno, V.M. Sanchez, M.J. Blommers, C. Griesinger, Derivation of dihedral angles from $\mathrm{CH}-\mathrm{CH}$ dipolar-dipolar cross-correlated relaxation rates: a $\mathrm{C}-\mathrm{C}$ torsion involving a quaternary carbon atom in epothilone $\mathrm{A}$ bound to tubulin, Angew. Chem. Int. Ed. Engl. 42 (2003) 2515-2517.

[4] G.M. Clore, A.M. Gronenborn, Theory of the time-dependent transferred nuclear Overhauser effect-applications to structural-analysis of ligand protein complexes in solution, J. Magn. Reson. 53 (1983) 423-442.

[5] G. Lippens, K. Hallenga, D. Vanbelle, S.J. Wodak, N.R. Nirmala, P. Hill, K.C. Russell, D.D. Smith, V.J. Hruby, Transfer nuclear Overhauser effect study of the conformation of oxytocin bound to bovine neurophysin-I, Biochemistry 32 (1993) 9423-9434.

[6] Q. Ning, D.R. Ripoll, Z. Szewczuk, Y. Konishi, F. Ni, Thrombin-bound conformation of a cyclic anticoagulant peptide using transferred nuclear Overhauser effect (NOE), distance geometry, and NOE simulations, Biopolymers 34 (1994) 1125-1137.

[7] J. Anglister, F. Naider, Nuclear-magnetic-resonance for studying peptide antibody complexes by transferred nuclear Overhauser effect difference spectroscopy, Methods Enzymol. 203 (1991) 228-241.

[8] C. Dalvit, G. Fogliatto, A. Stewart, M. Veronesi, B. Stockman, WaterLOGSY as a method for primary NMR screening: practical aspects and range of applicability, J. Biomol. NMR 21 (2001) 349-359.
[9] M. Mayer, B. Meyer, Mapping the active site of angiotensin-converting enzyme by transferred NOE spectroscopy, J. Med. Chem. 43 (2000) 2093-2099.

[10] M. Mayer, B. Meyer, Group epitope mapping by saturation transfer difference NMR to identify segments of a ligand in direct contact with a protein receptor, J. Am. Chem. Soc. 123 (2001) 6108-6117.

[11] J. Orts, S.K. Grimm, C. Griesinger, K.U. Wendt, S. Bartoschek, T. Carlomagno, Specific methyl group protonation for the measurement of pharmacophorespecific interligand NOE interactions, Chemistry 14 (2008) 7517-7520.

[12] J. Orts, J. Tuma, M. Reese, S.K. Grimm, P. Monecke, S. Bartoschek, A. Schiffer, K.U. Wendt, C. Griesinger, T. Carlomagno, Crystallography-independent determination of ligand binding modes, Angew. Chem. Int. Ed. Engl. 47 (2008) 7736-7740.

[13] M. Reese, V.M. Sanchez-Pedregal, K. Kubicek, J. Meiler, M.J. Blommers, C. Griesinger, T. Carlomagno, Structural basis of the activity of the microtubulestabilizing agent epothilone A studied by NMR spectroscopy in solution, Angew. Chem. Int. Ed. Engl. 46 (2007) 1864-1868.

[14] V.M. Sanchez-Pedregal, M. Reese, J. Meiler, M.J. Blommers, C. Griesinger, T. Carlomagno, The INPHARMA method: protein-mediated interligand NOEs for pharmacophore mapping, Angew. Chem. Int. Ed. Engl. 44 (2005) 4172-4175.

[15] R.W. Behling, T. Yamane, G. Navon, L.W. Jelinski, Conformation of acetylcholine bound to the nicotinic acetylcholine-receptor, Proc. Natl. Acad. Sci. USA 85 (1988) 6721-6725

[16] F. Ni, Y. Zhu, Accounting for ligand-protein interactions in the relaxationmatrix analysis of transferred nuclear Overhauser effects, J. Magn. Reson. B 103 (1994) 180-184.

[17] W. Lee, N.R. Krishna, Influence of conformational exchange on the 2d Noesy spectra of biomolecules existing in multiple conformations, J. Magn. Reson. 98 (1992) 36-48.

[18] G.M. Lippens, C. Cerf, K. Hallenga, Theory and experimental results of transferNoe experiments: 1 . The influence of the off rate versus cross-relaxation rates, J. Magn. Reson. 99 (1992) 268-281.

[19] S. Macura, R.R. Ernst, Elucidation of cross relaxation in liquids by twodimensional NMR spectroscopy, Mol. Phys. 100 (2002) 135-147 (Reprinted from Mol. Phys. 41 (1980) 95-117).

[20] J. Zheng, C.B. Post, Protein indirect relaxation effects in exchange-transferred Noesy by a rate-matrix analysis, J. Magn. Reson. B 101 (1993) 262-270.

[21] R.E. London, M.E. Perlman, D.G. Davis, Relaxation-matrix analysis of the transferred nuclear Overhauser effect for finite exchange-rates, J. Magn. Reson. 97 (1992) 79-98.

[22] N.R. Nirmala, G.M. Lippens, K. Hallenga, Theory and experimental results of transfer Noe experiments: 2 . The influence of residual mobility and relaxation centers inside the protein on the size of transfer Noes, J. Magn. Reson. 100 (1992) 25-42.

[23] P. Balaram, A.A. Bothner-By, E. Breslow, Localization of tyrosine at binding-site of neurophysin Ii by negative nuclear Overhauser effects, J. Am. Chem. Soc. 94 (1972) 4017-4018

[24] P. Balaram, A.A. Bothner-By, J. Dadok, Negative nuclear Overhauser effects as probes of macromolecular structure, J. Am. Chem. Soc. 94 (1972) 4015-4017.

[25] H.M. Edwards, Galois Theory, Springer-Verlag, 1984. ISBN 0-387-909080-X.

[26] I.E. Leonard, The matrix exponential, SIAM Rev. 38 (1996) 507-512.

[27] A.P. Campbell, B.D. Sykes, Effects of internal motions on the development of the two-dimensional transferred nuclear Overhauser effect, J. Biomol. NMR 1 (1991) 391-402.

[28] G. Lipari, A. Szabo, Model-free approach to the interpretation of nuclear magnetic-resonance relaxation in macromolecules: 1 . Theory and range of validity, J. Am. Chem. Soc. 104 (1982) 4546-4559. 